\title{
MicroRNA-199a induces differentiation of induced pluripotent stem cells into endothelial cells by targeting sirtuin 1
}

\author{
ZHOUBIN LI ${ }^{1}$, ANDRIANA MARGARITI ${ }^{2}$, YUTAO WU $^{3}$, FENG YANG $^{3}$, JIAN HU $^{1}$, \\ LI ZHANG ${ }^{3}$ and TING CHEN ${ }^{3}$
}

\begin{abstract}
${ }^{1}$ Department of Cardiothoracic Surgery, First Affiliated Hospital, School of Medicine, Zhejiang University, Hangzhou, Zhejiang 310016, P.R. China; ${ }^{2}$ Centre for Experimental Medicine, School of Medicine, Dentistry and Biomedical Sciences, Queen's University Belfast, Belfast BT12 6BA, UK; ${ }^{3}$ Department of Cardiology, First Affiliated Hospital, School of Medicine, Zhejiang University, Hangzhou, Zhejiang 310016, P.R. China
\end{abstract}

Received August 15, 2014; Accepted April 30, 2015

DOI: $10.3892 / \mathrm{mmr} .2015 .3845$

\begin{abstract}
The ability to reprogram induced pluripotent stem (iPS) cells from somatic cells may facilitate significant advances in regenerative medicine. MicroRNAs (miRNAs) are involved in a number of core biological processes, including cardiogenesis, hematopoietic lineage differentiation and oncogenesis. An improved understanding of the complex molecular signals that are required for the differentiation of iPS cells into endothelial cells (ECs) may allow specific targeting of their activity in order to enhance cell differentiation and promote tissue regeneration. The present study reports that miR-199a is involved in EC differentiation from iPS cells. Augmented expression of miR-199a was detected during EC differentiation, and reached higher levels during the later stages of this process. Furthermore, miR-199a inhibited the differentiation of iPS cells into smooth muscle cells. Notably, sirtuin 1 was identified as a target of miR-199a . Finally, the ability of miR-199a to induce angiogenesis was evaluated in vitro, using Matrigel plugs assays. This may indicate a novel function for miR-199a as a regulator of the phenotypic switch during vascular cell differentiation. The present study provides support to the notion that with an understanding of the molecular mechanisms underlying vascular cell differentiation, stem cell regenerative therapy may ultimately be developed as an effective treatment for cardiovascular disease.
\end{abstract}

\section{Introduction}

In 2006, Takahashi and Yamanaka (1) reported the direct reprogramming of murine embryonic fibroblasts (MEFs) into pluripotent stem cells, following the introduction of four

Correspondence to: Dr Ting Chen, Department of Cardiology, First Affiliated Hospital, School of Medicine, Zhejiang University, 79 Qingchun Road, Hangzhou, Zhejiang 310016, P.R. China

E-mail: ting010151452@aliyun.com

Key words: microRNA-199a, induced pluripotent stem cells, differentiation, endothelial cells, sirtuin 1 transcription factors (octamer-binding transcription factor 4, sex-determining region $\mathrm{Y}$ box-2, Kruppel-like factor 4 and cMyc). Subsequently, a number of laboratories have derived induced pluripotent stem cells from somatic cells. The identification of a method with which to reprogram induced pluripotent stem (iPS) cells from somatic cells (2) may facilitate advances in regenerative medicine. In contrast to embryonic stem cells, iPS cells may be administered without raising ethical or alloimmune concerns. iPS cells have exhibited the potential to differentiate into a number of cell lineages, including CD34+ progenitor cells (3), cardiomyocytes (4) and endothelial cells (ECs) (5). They have the capacity for unlimited growth and self-renewal, and are able to differentiate into all types of mature tissue cells. In recent years, accumulating evidence has indicated that iPS cells may differentiate into ECs in vitro or in vivo $(6,7)$.

ECs line the blood vessels of the circulatory system, and form the barrier between the circulating blood and the rest of the vessel wall. The endothelium is a dynamic and heterogeneous organ with secretory, metabolic, synthetic and immunological functions . Impaired EC function may result in hypertension, thrombosis, inflammation and atherosclerosis (8). Repair and regeneration of vascular cells, in particular of ECs, has been a research focus for a number of years. However, in the context of human disease, the use of adult progenitor or vascular stem cells has certain limitations, such as the identification and availability of appropriate, and effective cell-types (9). Recently, the ability to derive ECs from pluripotent stem cells has extended the scope of regenerative medicine $(10,11)$.

MiRNAs, an emerging class of highly conserved, non-coding small RNAs, regulate gene expression at the post-transcriptional level by inhibiting the translation of protein from mRNA or by promoting the degradation of mRNA. MiRNAs are involved in a number of core biological processes (12-15), including cardiogenesis, hematopoietic lineage differentiation and oncogenesis. Certain specific miRNAs that regulate endothelial cell functions and angiogenesis, have been described $(16,17)$. For example, Let7-f, miR-27b and miR-130a have been identified as proangiogenic miRNAs. Others, such as miR-221 and miR-222, have been shown to inhibit in vitro endothelial cell migration, proliferation and angiogenesis (16). 
SirT1 is a member of the a NAD ${ }^{+}$-dependent class III group of histone deacetylases and has been reported to be involved in a variety of biological systems and cellular functions (18). Recent data also suggests that SirT1 is a critical mediator in the regulation of various development genes during stem cell differentiation (19) and is important role in different cellular differentiations, including endothelial progenitor cells (20), hematopoietic cells (21) and osteoblasts $(22,23)$. Several previous studies have demonstrated the regulation of miRNAs in a myriad of vascular biological events. However, how they control EC fate commitment and the mechanisms involved in these differentiation processes remain to be elucidated. Therefore, the present study aimed to determine the possible roles of miRNAs in the differentiation processes in iPS cells.

\section{Materials and methods}

Materials. Cell culture media, serum and cell culture supplements were obtained from the American Type Culture Collection (ATCC; Manassas, VA, USA), Millipore, Invitrogen Life Technologies (Carlsbad, CA, USA) and PAA. Antibodies against rabbit anti-VE-cadherin (1:1,000; cat. no. Ab33168), and rabbit anti-SM22 (1:1,000; cat. no. ab14106) were obtained from Abcam. An antibody against mouse anti-smooth muscle actin (SMA; 1:1,000; cat. no. A5228) was purchased from Sigma-Aldrich (St. Louis, MO, USA). Antibodies against rabbit anti-Sirt1 (1:1,000; cat. no. sc-15404), goat anti-CD31 (1:500' cat. no. sc-1506) and rabbit anti-GAPDH (1:1,000; cat.no.sc-25778) were obtained from Santa Cruz Biotechnology, Inc. (Dallas, TX, USA). The secondary antibodies for western blotting were obtained from Dakocytomation and Abcam.

iPS cell culture and differentiation. Mouse iPS cells were generated in our laboratory, as previously described (24). Mouse iPS cells were cultured on gelatin-coated flasks (PBS containing $0.04 \%$ of gelatin from bovine skin; Sigma-Aldrich) in Dulbecco's modified Eagle's medium (DMEM; ATCC) supplemented with $10 \%$ fetal bovine serum, $100 \mathrm{IU} / \mathrm{ml}$ penicillin and $100 \mu \mathrm{g} / \mathrm{ml}$ streptomycin (Invitrogen Life Technologies); $10 \mathrm{ng} / \mathrm{ml} \mathrm{recom-}$ binant human leukemia inhibitory factor (Millipore); and $0.1 \mathrm{mM} / 0.05 \mathrm{mM}$ 2-mercaptoethanol (Invitrogen Life Technologies) in a humidified incubator, supplemented with $5 \% \mathrm{CO}_{2}$. Cells were passaged every 2 days at a ratio of 1:6. Differentiation of iPS cells was induced by seeding the cells on type IV mouse collagen $(5 \mu \mathrm{g} / \mathrm{ml})$-coated dishes in differentiation medium (DM) that contained $\alpha$-MEM supplemented with $10 \%$ FBS (Invitrogen Life Technologies), $0.05 \mathrm{~mm}$ 2-mercaptoethanol, 100 units $/ \mathrm{ml}$ penicillin, and $100 \mu \mathrm{g} / \mathrm{ml}$ streptomycin in the presence of $50 \mathrm{ng} / \mathrm{ml}$ vascular endothelial growth factor (VEGF) for the time points indicated.

miR-199a transient transfection. Manipulation of miR-199a levels in iPS cells that had differentiated with or without VEGF for 4 days, and cultured to $60-70 \%$ confluence, was performed using Pre-199b, or the non-targeting control (Pre-ctrl; Ambion AB). Inhibition of miR-199a was performed using the locked nucleic acid (LNA) inhibitor of miR-199a, LNA-199b, or a negative control (LNA-ctrl; Exiqon). All transfections were performed using Lipofectamine ${ }^{\mathrm{TM}}$ RNAiMAX (Invitrogen Life Technologies), at a final concen- tration of $50 \mathrm{nM}$, according to the manufacturer's instructions. In order to detect miRNA, total RNA was isolated using the miRVana miRNA isolation kit (Ambion), according to the manufacturer's instructions. Total and miRNA-specific cDNA was generated using the TaqMan ${ }^{\circledR}$ MicroRNA Reverse Transcription kit (Ambion), and miRVana quantitative RT-PCR primer sets for miRNAs (Ambion). The polymerase chain reaction $(\mathrm{PCR})$ reaction was directly monitored using the ABI PRISM 7000 Sequence Detection System (Applied Biosystems, CA). Briefly, cDNA was produced from enriched miRNA using the TaqMan MicroRNA RT kit. U6 RNA was used as an endogenous control.

Reverse transcription-PCR (RT-PCR). RT-PCR and quantitative PCR (qPCR) were performed as described previously (25). Total RNA was extracted using the RNeasy Mini kit (Qiagen, Shanghai, China), according to the manufacturer's instructions. Reverse transcription for RNA $(2 \mu \mathrm{g})$ was performed using an Improm-IITM RT kit (Promega, Madison, WI, USA) with RNase inhibitor (Promega), and Random primers (Promega). cDNA was analyzed by PCR using $20 \mathrm{ng}$ cDNA in a $50 \mu \mathrm{l}$ reaction volume containing primers and Ex-Taq DNA polymerase (Takara, Dalian, China). The PCR conditions were as follows: 32 cycles of $94^{\circ} \mathrm{C}$ for $60 \mathrm{sec}, 58^{\circ} \mathrm{C}$ for $60 \mathrm{sec}$ and $72^{\circ} \mathrm{C}$ for $90 \mathrm{sec}$.

$q P C R$. Relative gene expression was determined by qPCR, using $2 \mathrm{ng}$ of cDNA (relative to RNA amount) for each sample with the SYBR Green Master Mix in a $20-\mu 1$ reaction. Ct values were measured using an ABI Prism 7000 sequence detector (Applied Biosystems, Foster City, CA, USA). 18S ribosomal RNA was used as the endogenous control, against which the quantities of RNA in each sample were normalized. For each sample, PCR was performed in duplicate in a 96-well reaction plate (Eppendorf, twin. tec real time PCR plates). The gene was considered undetectable beyond 35 cycles. The following primer sets were used: Forward: 5'-CCAGTAAGTGCGGGTCATAA-3' and reverse: 5'-CCGAGGGCCTCACTAAACC-3' for $18 \mathrm{~S}$, forward: 5'-AAGAAACCGCTGATCGGCA-3' and reverse: 5'-TCGGAAGAATTGGCCTCTGTC-3' for VE-cadherin, forward: 5'-CAAACAGAAACCCGTGGAGAT-3' and reverse: 5'-ACCGTAATGGCTGTTGGCTTC-3' for CD31, forward: 5'-CGGGCAATTTCCATTGGCTTCTCA-3' and reverse: 5'-GGCCACACGAAGCTCGTTATAG-3' for SMA and forward: 5'-GATATGGCAGCAGTGCAGAG-3' and reverse: 5'-AGTTGGCTGTCTGTGAAGTC-3' for SM22.

Immunoblotting. Cells were harvested and washed with cold PBS, re-suspended in lysis buffer (25 mM Tris-Cl, $\mathrm{pH} 7.5$; $120 \mathrm{mM} \mathrm{NaCl} ; 1 \mathrm{mM}$ EDTA, pH 8.0; and 0.5\% Triton X100), supplemented with protease inhibitors (Sigma-Aldrich) and lysed by ultra-sonication (twice, for 6 sec each time; Bradson Sonifier 150; Bradson, St. Louis, MO, USA) in order to obtain whole cell lysates. The protein concentration was determined using the Biorad Protein Assay Reagent. Whole lysate $(50 \mu \mathrm{g})$ was applied to SDS-PAGE gels and transferred to Hybond PVDF membranes (Amersham Pharmacia Biotech, St. Albans, UK), followed by a standard western blotting procedure. The bound primary antibodies were detected by the use of horseradish 
A

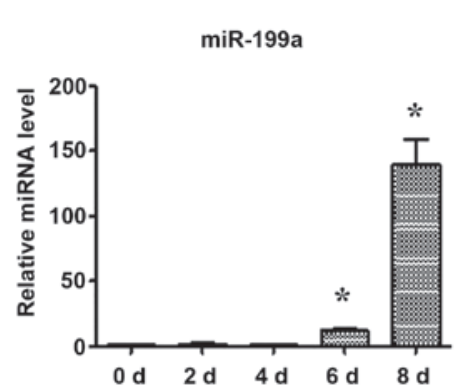

C

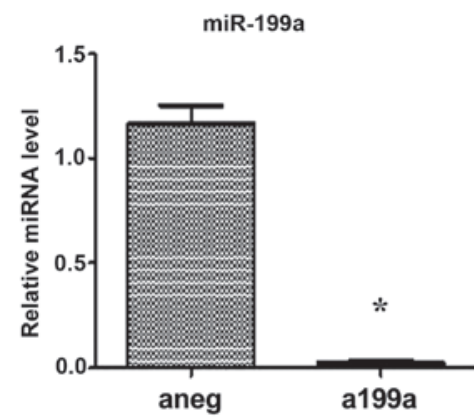

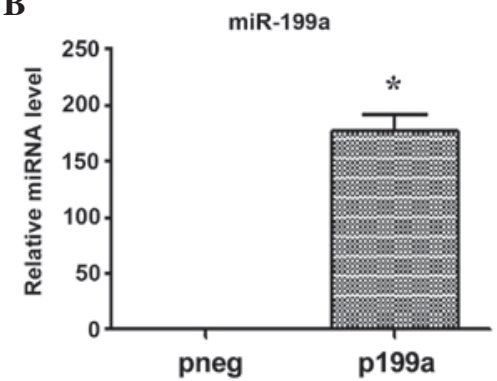

D

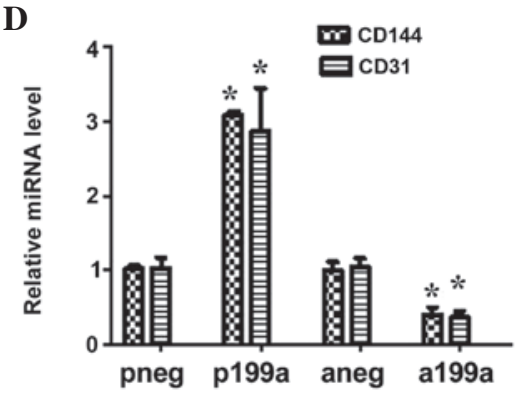

$\mathbf{E}$

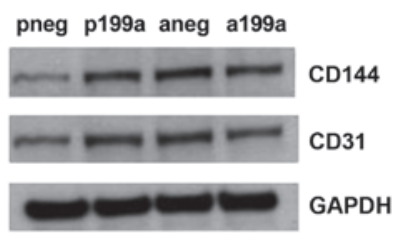

F

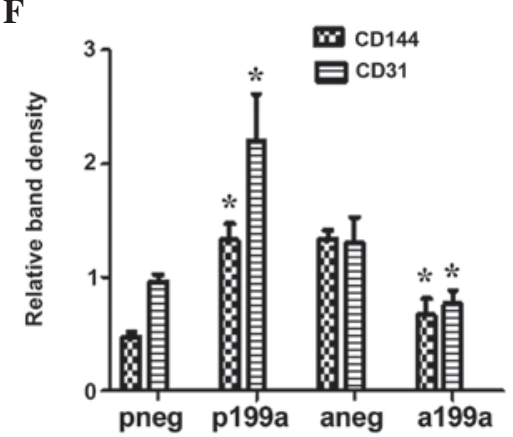

Figure 1. Effect of miR-199a in EC differentiation from iPS cells. Mouse iPS cells were seeded on Collagen IV-coated dishes and cultured in differentiated media supplemented with $50 \mathrm{ng} / \mathrm{ml}$ VEGF. (A) The expression of miR-199a was found to be upregulated during EC differentiation, as demonstrated by RT-qPCR. (B) iPS cells were forced to differentiate towards ECs for 4 days and then efficiently transfected with either miR-199a precursor molecules (premiR-199a) or an miR-199a inhibitor (anti-miR-199a), which resulted in significant miR-199a upregulation and repression, respectively. (C) On day 7, the cells were harvested and further RT-qPCR analysis demonstrated that miR-199a increased the mRNA (D) and protein (E) and (F) expression of the endothelial markers, CD144 and CD31. Data are presented as the mean \pm SEM. $n=3$. ${ }^{*} \mathrm{P}<0.05$. RT-qPCR, reverse transcription-quantitative polymerase chain reaction; SEM, standard error of the mean; miRNA, microRNA; EC, endothelial cell; iPS cells, induced pluripotent stem cells; VEGF, vascular endothelial growth factor; pneg, mimic negative control; P199a, miR-199b mimic; Aneg, inhibitor negative control; A199b, miR-199b inhibitor.

peroxidase-conjugated secondary antibody and the ECL detection system (GE Health). The band density was semiquantified using ImageJ v 1.45S software (NIH, Bethseda, MA, USA).

Luciferase reporter assay. For the luciferase reporter assays, $3 \times 10^{4}$ iPS cells were seeded into collagen-coated wells in a 12-well plate in differentiated media (DM). After $72 \mathrm{~h}$, cells were transfected with luciferase plasmids under the control of the Sirt1 3'UTR Lenti-reporter-Luc Vector (ABM), in addition to the Pre-199b, 199b inhibitor and controls. Briefly, $0.33 \mu \mathrm{g} / \mathrm{well}$ of the reporter plasmids were cotransfected with the Pre-199b, 199b inhibitor and controls (2 $\mu \mathrm{l} /$ well $)$ using jetPRIME $^{\circledR}$ (Polyplus-transfection SA), according to the manufacturer's instructions. pGL3-Luc Renilla $(0.1 \mu \mathrm{g} /$ well) was included in all transfection assays as an internal control. Luciferase and Renilla (Promega Corporation) activity assays were detected at $48 \mathrm{~h}$ following transfection using a standard protocol (2). The relative luciferase unit (RLU) was defined as the ratio of luciferase activity to Renilla activity, with that of the control set as 1.0.

In vitro tube formation assay. iPS cells were differentiated in the absence of VEGF for 4 days, and then transfected with Pre-199a or Pre-ctrl. In vitro angiogenesis assays were conducted after $48 \mathrm{~h}$, as described previously $(2,24)$. Cell suspensions, containing $4 \times 10^{4}$ transfected cells, were placed on top of the $50 \mu \mathrm{l} /$ well Matrigel $(10 \mathrm{mg} / \mathrm{ml}$; BD Matrigel Basement Membrane Matrix, A6661; BD Biosciences, Franklin Lakes, NJ, USA) in 8-well chamber slides (BD Biosciences). Rearrangement of cells and the formation of capillary-like structures were observed at 6-12 h.

Statistical analysis. Data are expressed as the mean \pm standard error of the mean, and were analyzed using GraphPad Prism 5 
A

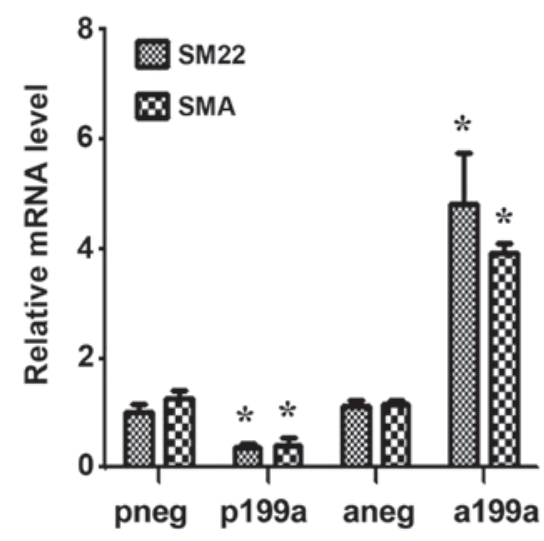

B

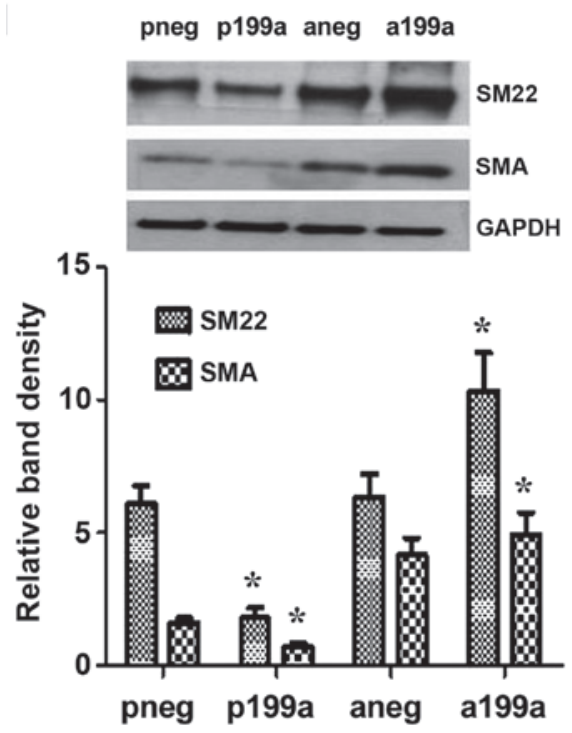

Figure 2. miR-199a may act as a phenotypic switch in vascular cell differentiation. iPS cells were seeded on collagen IV-coated dishes, and cultured with differentiated media in the absence of VEGF for 4 days. They were then forced to differentiate towards vascular progenitor cells. (A) Overexpression of miR-199a suppressed the mRNA (A) and protein (B) expression of the SMC markers, SMA and SM22, as demonstrated by reverse transcription-quantitative polymerase chain reaction and western blotting, respectively. The miR-199a inhibitor favored the induction of the SMC markers. Data are presented as the mean \pm SEM. $n=3$. ${ }^{*} \mathrm{P}<0.05$. miRNA, microRNA; iPS cell, induced pluripotent stem cell; VEGF, vascular endothelial growth factor; SMC, smooth muscle cell; pneg, mimic negative control; P199a, miR-199b mimic; Aneg, inhibitor negative control; A199b, miR-199b inhibitor.

software (GraphPad Software, Inc., La Jolla, CA, USA) with two-tailed Student's t-test for two groups or pairwise comparisons. $\mathrm{P}<0.05$ was considered to indicate a statistically significant difference.

\section{Results}

MiR-199a is involved in EC differentiation from iPS cells. The expression of miR-199a was found to be upregulated during EC differentiation from iPS cells on days 0 to 8 (Fig. 1A). In order to examine the effect of miR-199a on EC differentiation, a chemically synthesized miR-199a inhibitor or Pre-199b (mimic) were administered. iPS cells were forced to differentiate towards ECs for 4 days and were efficiently transfected with either miR-199a precursor molecules (pre-miR-199a)
A
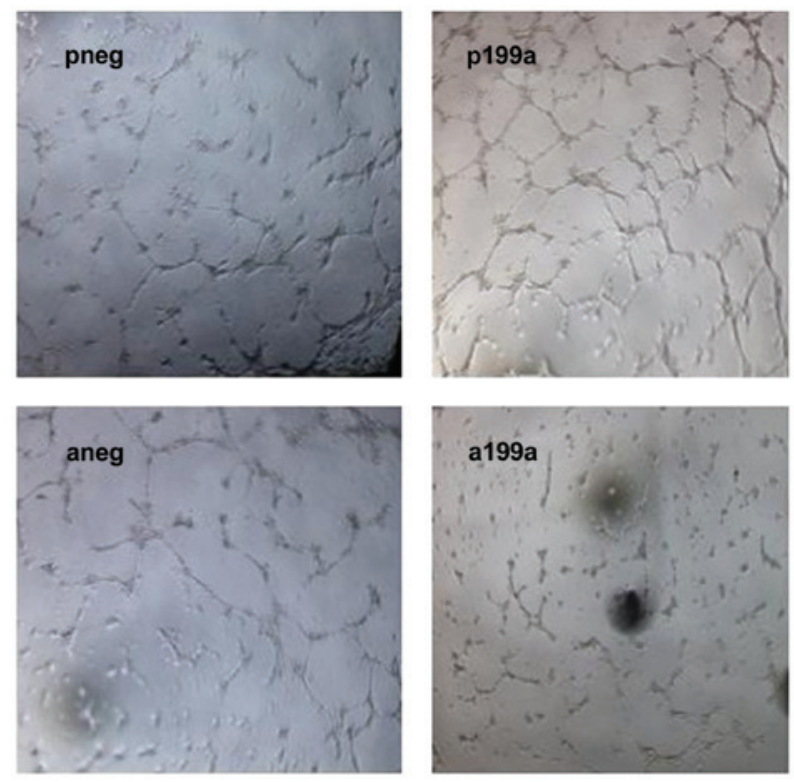

B

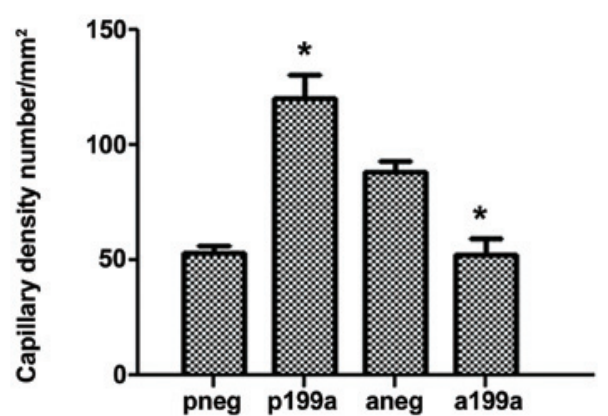

Figure 3. miR-199a induced angiogenesis in vitro. iPS cells were seeded on collagen IV-coated plates and cultured in differentiated media in the absence of VEGF for 4 days. Then the miR-199a mimic or control were introduced to the cells by transfection. After 2 days, the cells were subjected to Matrigel assays plugs in vitro. (A) Upper panel: miR-199a formed vascular structures within a 4-6 h in the in vitro Matrigel assays, compared with the control where less defined vascular structures were observed. Lower panel: Additional experiments were performed, using the miR-199a inhibitor. The miR-199a inhibitor suppressed the formation of vascular structures in the in vitro Matrigel plugs assays, compared with the control cells. (B) Quantification of results in (A). Data are presented as the mean \pm SEM. $n=3$. ${ }^{*} \mathrm{P}<0.05$. miRNA, microRNA; iPS cell, induced pluripotent stem cell; VEGF, vascular endothelial growth factor; pneg, mimic negative control; P199a, miR-199b mimic; Aneg, inhibitor negative control; A199b, miR-199b inhibitor.

or an miR-199a inhibitor (anti-miR-199a), resulting in significant miR-199a upregulation and repression, respectively (Fig. 1B and C). Furthermore, the miR-199a mimic increased the mRNA (Fig. 1D) and protein (Fig. 1E and F) expression of the endothelial markers, CD144 and CD31. By contrast, the miR-199a inhibitor decreased the expression of EC markers. These initial observations suggest the involvement of miR-199a in EC differentiation from iPS cells.

MiR-199a acts as a phenotypic switch in vascular cell differentiation. Subsequent experiments demonstrated that miR-199a concomitantly suppressed the mRNA and protein expression of the smooth muscle cells (SMC) markers, SMA and SM22, when iPS cells were induced to differentiate towards vascular progenitor cells, by seeding the cells on Collagen IV and culturing them in DM in the absence of VEGF. By contrast, 
A

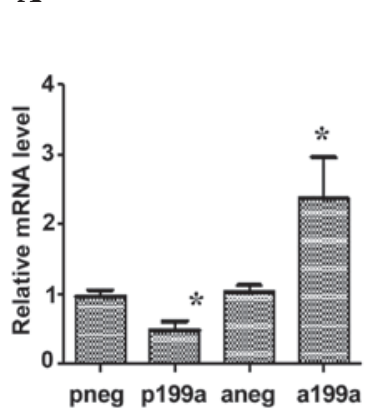

B

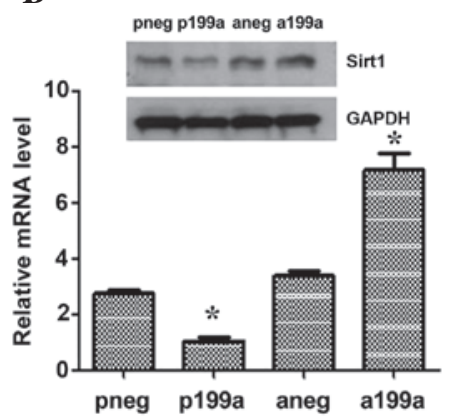

\section{C}

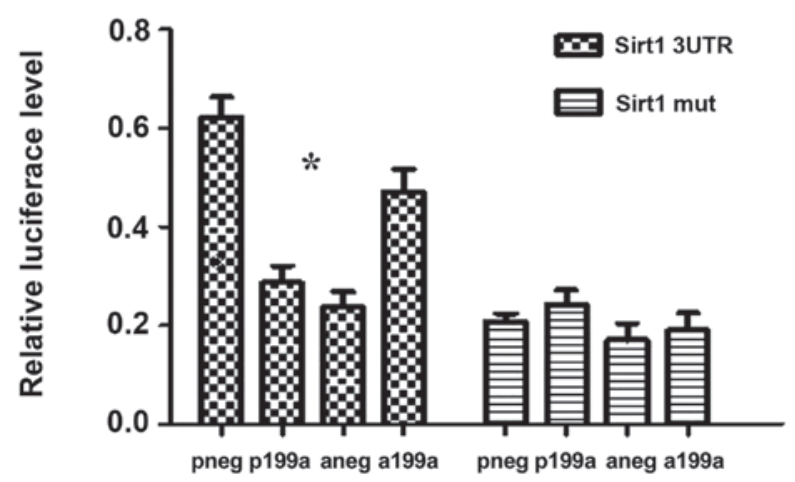

Figure 4. miR-199a targeted Sirt1. miR-199a suppressed the mRNA (A) and protein (B) expression of Sirt1 during EC differentiation derived from iPS cells. The miR-199a inhibitor induced the expression of Sirt1. (C) iPS cells were forced to differentiate towards ECs for 4 days. Cotransfection of miR199a or inhibition with the luciferase plasmid of the 3'UTR of Sirt1 was then performed. After $48 \mathrm{~h}$, the cells were subjected to luciferase analysis, which demonstrated that the 3'UTR of Sirt1 is a direct target for miR-199a. Data are presented as the mean \pm SEM. $n=3$. ${ }^{*} \mathrm{P}<0.05$. miRNA, microRNA; Sirt1, sirtuin 1; EC, endothelial cell; iPS cell, induced pluripotent stem cell; UTR, untranslated region; pneg, mimic negative control; P199a, miR-199b mimic; Aneg, inhibitor negative control; A199b, miR-199b inhibitor.

the miR-199a inhibitor favored the induction of the SMC markers (Fig. 2A and B). These results indicate an association between miR-199a and SMC differentiation, and it is hypothesized that miR-199a may act as a phenotypic switch during vascular cell differentiation.

MiR-199a induces angiogenesis in vitro. In order to evaluate the role of miR-199a in angiogenesis, the levels of miR-199a were modulated either by overexpression using an miR-199a mimic, or by knockdown using an miR-199a inhibitor. In brief, mouse iPS cells were seeded on collagen IV-coated plates and cultured in DM in the absence of VEGF. The miR-199a mimic or control were then introduced to the cells by transfection. After 4 days, the cells were subjected to Matrigel assays plugs in vitro. The results demonstrated that in the presence of the miR-199a mimic, the differentiated cells had formed vascular structures within a few hours, in comparison with the control cells, in which where few or no defined vascular structures were observed (Fig. 3A and B). Further experiments were performed in the presence of the miR-199a inhibitor. These cells were then subjected to Matrigel plugs assays in vitro. The results demonstrated that the formation of vascular structures was suppressed in cells treated with the miR-199a inhibitor, in comparison with the control cells.

MiR-199a targets Sirt1. In order to further investigate the mechanisms underlying the regulation of EC differentiation by miR-199a, potential mRNA targets of miR-199a were scrutinized. Sirtl emerged as one of the primary targets of miR-199a in several computational algorithmic databases, including Targetscan (www.targetscan.org), pictar (www.pictar. mdc-berlin.de) iand miRanda (www.microrna.org), and two highly conserved binding sites for miR-199a have also previously been identified within the Sirt1 3'UTR (26). The results of the present study supported this hypothesis, as the Sirt1 gene and protein expression was significantly downregulated or upregulated following overexpression or inhibition of miR-199a in the differentiating iPS cells (Fig. 4A and B), respectively. This indicated that Sirt1 is regulated, directly or indirectly, by miR-199a. In order to investigate this possibility, the 3'UTR of Sirt1, which contained the binding sites for miR-199a, was cloned into a luciferase reporter. The results from the miRNA reporter assay demonstrated that the activity of luciferase from the construct, containing the Sirt1 3'UTR, was significantly downregulated following miR-199a overexpression (Fig. 4C). This data has provides clear evidence that Sirt1 is a target of miR-199a.

\section{Discussion}

iPS-derived ECs may be used to treat damaged vessels, to avoid restenosis and to develop tissue-engineered vascular grafts, which are significant challenges for regenerative medicine (27). To date, studies have demonstrated the regulation by miRNAs of a myriad of vascular biological events (28). By studying the non-coding RNAs and their regulatory signalling, it is possible to improve understanding of the molecular mechanisms that govern cell fate specification. The present study, aimed to elucidate the molecular mechanisms underlying the differentiation of iPS cells into ECs, and to identify miRNAs and their respective targets involved in this process, in order to aid the development of novel strategies for the treatment of vascular diseases, such as atherosclerosis; for the enhancement of neovascularization following ischemia; and for the prevention of atherosclerotic inflammation.

miR-199a is well-conserved in different species (29), and has been identified by diverse high-throughput screenings in a number of animal models of disease. The functions of miR-199a vary between systems. For example, miR-199a is involved in cardiomyocyte protection by rapid downregulation under hypoxic conditions and prompts hypoxia inducible factor $1 \alpha$ (HIF1a) expression (30). miR-199a has also been shown to be involved in stem cell differentiation (29), with differences in the expression of miR-199a detected prior to and following stem cell differentiation. For example, following the differentiation of human embryonic stem cells into pancreatic islet-like cells, miR-199a expression was found to be upregulated (31). Studies in ovarian cancer stem cells have shown that the two subtypes of epithelial ovarian cancer stem cells, exhibit distinct expression patterns of miR-199a (32). However, there is insufficient evidence to demonstrate how miR-199a controls commitment to EC differentiation or the mechanisms involved 
in these processes. To the best of our knowledge, the current study reports for the first time, that miR-199a is involved in EC differentiation from iPS cells. The expression of miR-199a was shown to be upregulated during EC differentiation, and an abundant expression of miR-199a was detected in the later stages of this process. Notably, miR-199a was demonstrated to target Sirt1. miR-199a has already been described as a potential regulator of Sirt1, in two independent studies. In the first, miR-199a was shown to promote migration and tube formation of human cytomegalovirus-infected endothelial cells via the downregulation of Sirt1 and eNOS (33). The second study found that downregulation of miR-199a repressed HIF1a and Sirt1 expression, and reinforced hypoxia preconditioning in cardiac myocytes (34). In accordance with these previous findings, the present study provides clear evidence that Sirt1 is an mRNA target of miR-199a and that miR-199a negatively regulates this target gene during EC differentiation from iPS cells. This hypothesis is supported by several lines of experimentation. Firstly, Sirt1 gene and protein expression levels were negatively regulated by miR-199a, as demonstrated in the experiments involving miR-199a overexpression and inhibition. Secondly, overexpression of miR-199a significantly upregulated Sirt1 3'UTR activity, while this upregulation was abrogated when the miR-199a binding sites were mutated.

Notably, in the present study, miR-199a exhibited the potential to induce angiogenesis, using Matrigel plug assays. In previous studies, ECs derived from pluripotent stem cells were incorporated into the microvasculature of ischemic tissue, thereby enhancing perfusion and improving function $(35,36)$. Currently, the potential of iPS cells to differentiate towards therapeutic cells is based on directed empiricism, while they are entirely dependent on combinations of growth factors, media and matrices to favor differentiation into the desired lineage. With regard to vascular regeneration, it is important to gain an improved understanding of epigenetic alterations, transcriptional activity and microRNA patterns associated with the differentiation processes, in order to efficiently generate therapeutic cells. Refined experimental protocols are required to reliably guide iPS cells to a vascular lineage $(37,38)$. The bioengineered vessels derived from these vascular cells, and their incorporation of into bypass grafts or stents, are anticipated future directions in stem cell research (39-41).

In addition, inhibition of miR-199a resulted in the robust induction of SMC marker expression. In 2007, Ferreira et al (42) reported that vascular progenitor cells isolated from human embryonic stem cells, gave rise to EC- and SMC-like cells, and formed vascular networks in vivo, providing strong evidences that ECs and SMCs are derived from a common progenitor. Therefore, it is possible that a molecular switch may regulate vascular cell differentiation, and may maintain the balance of these two cell lineages during development, disease-repair and cell differentiation. Therefore, the present findings may suggest a unique role miR-199a as a vascular cell phenotypic switch.

In conclusion, the present study has demonstrated a novel role for miR-199a in the differentiation of ECs from iPS cells, and has provided strong evidence that miR-199a negatively regulates its target gene, Sirt1, during EC differentiation. Furthermore, the current data support the hypothesis that miR-199a inhibits the differentiation of iPS cells into SMCs, which indicates that miR-199a may act as a potential regulator of the phenotypic switch during vascular cell differentiation. Finally, the induction of angiogenesis by miR-199a, was evaluated using Matrigel plug assays. These findings significantly increase the current understanding of the molecular mechanisms underlying EC differentiation, and may benefit future research into regenerative medicine and aid the development of the clinical application of this therapy.

\section{Acknowledgements}

This study was supported by the Zhejiang Provincial Natural Science Foundation of China (grant no. LQ14H020001) and the National Natural Science Foundation of China (grant no. 81373161/H1006).

\section{References}

1. Takahashi $\mathrm{K}$ and Yamanaka $\mathrm{S}$ : Induction of pluripotent stem cells from mouse embryonic and adult fibroblast cultures by defined factors. Cell 126: 663-676, 2006.

2. Margariti A, Winkler B, Karamariti E, Zampetaki A, Tsai TN, Baban D, Ragoussis J, Huang Y, Han JD, Zeng L, et al: Direct reprogramming of fibroblasts into endothelial cells capable of angiogenesis and reendothelialization in tissue-engineered vessels. Proc Natl Acad Sci USA 109: 13793-13798, 2012.

3. Park SW, Jun Koh Y, Jeon J, Cho YH, Jang MJ, Kang Y, Kim MJ, Choi C, Sook Cho Y, et al: Efficient differentiation of human pluripotent stem cells into functional CD34+ progenitor cells by combined modulation of the MEK/ERK and BMP4 signaling pathways. Blood 116: 5762-5772, 2010.

4. Dambrot C, Passier R, Atsma D and Mummery CL: Cardiomyocyte differentiation of pluripotent stem cells and their use as cardiac disease models. Biochem J 434: 25-35, 2011.

5. Li Z, Hu S, Ghosh Z, Han Z and Wu JC: Functional characterization and expression profiling of human induced pluripotent stem cell- and embryonic stem cell-derived endothelial cells. Stem Cells Dev 20: 1701-1710, 2011.

6. Rufaihah AJ, Huang NF, Kim J, Herold J, Volz KS, Park TS Lee JC, Zambidis ET, Reijo-Pera R and Cooke JP: Human induced pluripotent stem cell-derived endothelial cells exhibit functional heterogeneity. Am J Transl Res 5: 21-35, 2013.

7. Wong WT, Huang NF, Botham CM, Sayed N and Cooke JP: Endothelial cells derived from nuclear reprogramming. Circ Res 111: 1363-1375, 2012.

8. Otsuka F, Finn AV, Yazdani SK, Nakano M, Kolodgie FD and Virmani R: The importance of the endothelium in atherothrombosis and coronary stenting. Nat Rev Cardiol 9: 439-453, 2012.

9. Collado M, Blasco MA and Serrano M: Cellular senescence in cancer and aging. Cell 130: 223-233, 2007.

10. Cheng Y, Ji R, Yue J, Yang J, Liu X, Chen H, Dean DB and Zhang C: MicroRNAs are aberrantly expressed in hypertrophic heart: Do they play a role in cardiac hypertrophy? Am J Pathol 170: 1831-1840, 2007.

11. Sayed D, Hong C, Chen IY, Lypowy J and Abdellatif M: MicroRNAs play an essential role in the development of cardiac hypertrophy. Circ Res 100 416-424, 2007.

12. Chen T, Huang Z, Wang L, Wang Y, Wu F, Meng S and Wang C: MicroRNA-125a-5p partly regulates the inflammatory response, lipid uptake, and ORP9 expression in oxLDL-stimulated monocyte/macrophages. Cardiovasc Res 83: 131-139, 2009.

13. Chen T, Li Z, Jing T, Zhu W, Ge J, Zheng X, Pan X, Yan H and Zhu J: MicroRNA-146a regulates the maturation process and pro-inflammatory cytokine secretion by targeting CD40L in oxLDL-stimulated dendritic cells. FEBS Lett 585: 567-573, 2011.

14. Chen T, Li Z, Tu J, Zhu W, Ge J, Zheng X, Yang L, Pan X, Yan H and Zhu J: MicroRNA-29a regulates pro-inflammatory cytokine secretion and scavenger receptor expression by targeting LPL in oxLDL-stimulated dendritic cells. FEBS Lett 585: 657-663, 2011. 
15. Chen T, Yan H, Li Z, Jing T, Zhu W, Ge J, Zheng X, Pan X and Zhu J: MicroRNA-155 regulates lipid uptake, adhesion/chemokine marker secretion and SCG2 expression in oxLDL-stimulated dendritic cells/macrophages. Int J Cardiol 147: 446-447, 2011.

16. Kuehbacher A, Urbich C, Zeiher AM and Dimmeler S: Role of Dicer and Drosha for endothelial microRNA expression and angiogenesis. Circ Res 101: 59-68, 2007.

17. Urbich C, Kuehbacher A and Dimmeler S: Role of microRNAs in vascular diseases, inflammation, and angiogenesis. Cardiovasc Res 79: 581-588, 2008.

18. Yang Y, Duan W, Li Y, Jin Z, Yan J, Yu S and Yi D: Novel Role of Silent Information Regulator 1 in Myocardial Ischemia. Circulation 128: 2232-2240, 2013.

19. Calvanese V, Lara E, Suárez-Alvarez B, et al: Sirtuin 1 regulation of developmental genes during differentiation of stem cells. Proc Natl Acad Sci USA 107: 13736-13741, 2010.

20. Cheng BB, Yan ZQ, Yao QP, et al: Association of SIRT1 expression with shear stress induced endothelial progenitor cell differentiation. J Cell Biochem 113: 3663-3671, 2012.

21. Ou X, Chae HD, Wang RH, et al: SIRT1 deficiency compromises mouse embryonic stem cell hematopoietic differentiation, and embryonic and adult hematopoiesis in the mouse. Blood 117: 440-450, 2011.

22. Shakibaei M, Shayan P, Busch F, et al: Resveratrol mediated modulation of Sirt-1/Runx 2 promotes osteogenic differentiation of mesenchymal stem cells: potential role of Runx 2 deacetylation. PLoS One 7: e35712, 2012.

23. Srivastava S, Bedi U and Roy P. Synergistic actions of insulinsensitive and Sirtl-mediated pathways in the differentiation of mouse embryonic stem cells to osteoblast. Mol Cell Endocrinol 361: 153-164, 2012.

24. Di Bernardini E, Campagnolo P, Margariti A, Zampetaki A, Karamariti E, Hu Y and Xu Q: Endothelial lineage differentiation from induced pluripotent stem cells is regulated by microRNA-21 and transforming growth factor $\beta 2$ (TGF- $\beta 2$ ) pathways. J Biol Chem 289: 3383-3393, 2014

25. Margariti A, Zampetaki A, Xiao Q, Zhou B, Karamariti E, Martin D, Yin X, Mayr M, Li H, Zhang Z, et al: Histone deacetylase 7 controls endothelial cell growth through modulation of beta-catenin. Circ Res 106: 1202-1211, 2010

26. Yu X, Zhang L, Wen G, Zhao H, Luong LA, Chen Q, Huang Y, Zhu J, Ye S, Xu Q, Wang W and Xiao Q: Upregulated sirtuin 1 by miRNA-34a is required for smooth muscle cell differentiation from pluripotent stem cells. Cell Death Differ 19: 2014.

27. Hibino N, Duncan DR, Nalbandian A, Yi T, Qyang Y, Shinoka T and Breuer CK: Evaluation of the use of an induced puripotent stem cell sheet for the construction of tissue-engineered vascular grafts. J Thorac Cardiovasc Surg 143: 696-703, 2012.

28. Cai X, Hagedorn $\mathrm{CH}$ and Cullen BR. Human microRNAs are processed from capped, polyadenylated transcripts that can also function as mRNAs. RNA 10: 1957-1966, 2004.

29. Gu S and Chan WY: Flexible and versatile as a chameleon - sophisticated functions of microRNA-199a. Int J Mol Sci 13: 8449-8466, 2012.
30. Joshi HP, Subramanian IV, Schnettler EK, Ghosh G, Rupaimoole R, Evans C, Saluja M, Jing Y, Cristina I, Roy S, et al: Dynamin 2 along with microRNA-199a reciprocally regulate hypoxia-inducible factors and ovarian cancer metastasis. Proc Natl Acad Sci USA 111: 5331-5336, 2014.

31. Chen BZ, Yu SL, Singh S, Kao LP, Tsai ZY, Yang PC, Chen BH and Shoei-Lung Li S: Identification of microRNAs expressed highly in pancreatic islet-like cell clusters differentiated from human embryonic stem cells. Cell Biol Int 35: 29-37, 2011.

32. Yin G, Chen R, Alvero AB, Fu HH, Holmberg J, Glackin C, Rutherford T and Mor G: TWISTing stemness, inflammation and proliferation of epithelial ovarian cancer cells through MIR199A2/214. Oncogene 29: 3545-3553, 2010.

33. Zhang S, Liu L, Wang R, Tuo H, Guo Y, Yi L, Wang J and Wang D: MiR-199a-5p promotes migration and tube formation of human cytomegalovirus-infected endothelial cells through downregulation of SIRT1 and eNOS. Arch Virol 158: 2443-2452, 2013.

34. Rane S, He M, Sayed D, Vashistha H, Malhotra A, Sadoshima J, Vatner DE, Vatner SF and Abdellatif M: Downregulation of miR-199a derepresses hypoxia-inducible factor-1alpha and Sirtuin 1 and recapitulates hypoxia preconditioning in cardiac myocytes. Circ Res 104: 879-886, 2009.

35. Li Z, Wu JC, Sheikh AY, Kraft D, Cao F, Xie X, Patel M, Gambhir SS, Robbins RC, et al: Differentiation, survival, and function of embryonic stem cell derived endothelial cells for ischemic heart disease, Circulation 116 (Suppl 11): I46-I54, 2007.

36. Yamahara K, Sone M, Itoh H, Yamashita JK, Yurugi-Kobayashi T, Homma K, Chao TH, Miyashita K, Park K, Oyamada N, et al: Augmentation of neovascularization [corrected] in hindlimb ischemia by combined transplantation of human embryonic stem cells-derived endothelial and mural cells. PloS One 3: e1666, 2008.

37. Mauritz C, Schwanke K, Reppel M, Neef S, Katsirntaki K, Maier LS, Nguemo F, Menke S, Haustein M, Hescheler J, et al: Generation of functional murine cardiac myocytes from induced pluripotent stem cells. Circulation 118: 507-517, 2008.

38. Nishikawa S, Goldstein RA and Nierras CR: The promise of human induced pluripotent stem cells for research and therapy. Nat Rev Mol Cell Biol 9:725-729, 2008.

39. Asahara $\mathrm{T}$ and Kawamoto A: Endothelial progenitor cells for postnatal vasculogenesis. Am J Physiol Cell Physiol 287: C572-C579, 2004

40. Zhao R and Daley GQ: From fibroblasts to iPS cells: Induced pluripotency by defined factors. J Cell Biochem 105: 949-955, 2008.

41. Zhang L, Zhou J, Lu Q, Wei Y and Hu S: A novel small-diameter vascular graft: In vivo behavior of biodegradable three-layered tubular scaffolds. Biotechnol Bioeng 99: 1007-1015, 2008

42. Ferreira LS, Gerecht S, Shieh HF, Watson N, Rupnick MA, Dallabrida SM, Vunjak-Novakovic G and Langer R: Vascular progenitor cells isolated from human embryonic stem cells give rise to endothelial and smooth muscle like cells and form vascular networks in vivo. Circ Res 101: 286-294, 2007. 